\title{
The biharmonic homotopy problem for unit vector fields on 2-tori
}

\author{
E. Loubeau ${ }^{1} \cdot$ M. Markellos ${ }^{2}$
}

Received: 21 September 2018 / Accepted: 5 February 2019 / Published online: 15 February 2019 (c) Fondazione Annali di Matematica Pura ed Applicata and Springer-Verlag GmbH Germany, part of Springer Nature 2019

\begin{abstract}
The bienergy of smooth maps between Riemannian manifolds, when restricted to unit vector fields, yields two different variational problems depending on whether one takes the full functional or just the vertical contribution. Their critical points, called biharmonic unit vector fields and biharmonic unit sections, form different sets. Working with surfaces, we first obtain general characterizations of biharmonic unit vector fields and biharmonic unit sections under conformal change of the metric. In the case of a two-dimensional torus, this leads to a proof that biharmonic unit sections are always harmonic and a general existence theorem, in each homotopy class, for biharmonic unit vector fields.
\end{abstract}

Keywords Bienergy functional · Biharmonic unit vector fields · Biharmonic unit sections . 2-Torus

Mathematics Subject Classification Primary 58E20 - Secondary 53C20

\section{Introduction}

One of the most studied objects in Differential Geometry is the energy functional of a map $\varphi:(M, g) \rightarrow(N, h)$ between Riemannian manifolds, given by

$$
E_{1}(\varphi)=\frac{1}{2} \int_{M}\|\mathrm{~d} \varphi\|^{2} v_{g}
$$

where $\mathrm{d} \varphi$ denotes the differential of the $\operatorname{map} \varphi([1,4])$. Its critical points are called harmonic maps and are characterized by the vanishing of the tension field $\tau_{1}(\varphi)=\operatorname{tr} \nabla \mathrm{d} \varphi$.

$\bowtie$ E. Loubeau

Eric.Loubeau@univ-brest.fr

M. Markellos

mmarkellos@hotmail.gr

1 Département de Mathématiques, LMBA, UMR 6205, Université de Bretagne Occidentale, 6, Avenue Victor Le Gorgeu, CS 93837, 29238 Brest Cedex 3, France

2 Department of Mathematics and Statistics, University of Cyprus, P.O. Box 20537, 1678 Nicosia, Cyprus 
A unit vector field $V$ on $(M, g)$ is a section of the unit tangent sphere bundle $T_{1} M$, i.e. $V:(M, g) \rightarrow\left(T_{1} M, g_{S}\right)$, where $T_{1} M$ carries the Sasaki metric $g_{S}$ induced from $g$. Its energy $E_{1}(V)$ (resp. vertical energy $E_{1}^{v}(V)$ ) is given by

$$
E_{1}(V)=\frac{m}{2} \operatorname{vol}(M)+\frac{1}{2} \int_{M}\|\nabla V\|^{2} v_{g}, E_{1}^{v}(V)=\frac{1}{2} \int_{M}\|\nabla V\|^{2} v_{g},
$$

where $m=\operatorname{dim} M$ [11]. Critical points of $E_{1}$, respectively $E_{1}^{v}$, with respect to variations through unit vector fields are called harmonic unit vector fields, respectively harmonic unit sections. While both functionals clearly give the same critical points, this will no longer be the case for our problem. The corresponding critical point conditions have been determined in $[11,12]$.

In [8], Markellos and Urakawa defined the bienergy of a vector field $X$ on a Riemannian manifold $(M, g)$ as the $L^{2}$-norm of the tension field of the corresponding map $X:(M, g) \rightarrow$ $\left(T M, g_{S}\right)$. More precisely, they proved that if $(M, g)$ is compact and $X$ is biharmonic map, then $X$ must be parallel. Furthermore, this remains the case under the less stringent and arguably more natural condition that $X$ is a biharmonic vector field, i.e. a critical point of the bienergy functional with respect to variations through vector fields. The situation is very different when we consider the set of unit vector fields on $(M, g)$. The bienergy (resp. vertical bienergy) of unit vector field $V$ is defined by

$$
\begin{aligned}
& E_{2}(V)=\frac{1}{2} \int_{M}\left\|\tau_{1}(V)\right\|^{2} v_{g}=\frac{1}{2} \int_{M}\left\{g(S(V), S(V))+g(\bar{\Delta} V, \bar{\Delta} V)-(g(\bar{\Delta} V, V))^{2}\right\} v_{g}, \\
& E_{2}^{v}(V)=\frac{1}{2} \int_{M}\left\|\tau_{1}^{v}(V)\right\|^{2} v_{g}=\frac{1}{2} \int_{M}\left\{g(\bar{\Delta} V, \bar{\Delta} V)-(g(\bar{\Delta} V, V))^{2}\right\} v_{g},
\end{aligned}
$$

where $S(V)=\operatorname{tr} R(\nabla . V, V)$. and $\bar{\Delta} V=-\operatorname{tr} \nabla^{2} V$. Critical points of $E_{2}$ (resp. $E_{2}^{v}$ ) with respect to variations through unit sections are called biharmonic unit vector fields (resp. biharmonic unit sections).

In this paper, we address the following questions:

Question 1.1 Do there exist examples of biharmonic unit sections?

Question 1.2 Is there a relation between the notions of "harmonic unit section", a "biharmonic unit section" and "biharmonic unit vector field"?

Question 1.3 Do biharmonic unit sections and biharmonic unit vector fields exist on a twodimensional torus?

We should point out that a harmonic unit section is automatically a biharmonic unit section. In contrast with the harmonic case, we show that the notions of "biharmonic unit vector field" and "biharmonic unit section" are not equivalent (see Remark 2.1). To answer Question 1.3, we show that biharmonic unit sections and biharmonic unit vector fields are not invariant under conformal change of the metric on a 2-torus. This is a significant difference with the set of harmonic unit vector fields on $\left(T^{2}, g\right)$ which is conformally invariant ([11]).

We refer to [8] for the geometry of the tangent bundle.

Definition 1.1 Let $(M, g)$ be a Riemannian manifold. A unit vector field $V \in \mathfrak{X}^{1}(M)$ is a biharmonic unit vector field (resp. biharmonic unit section) if the corresponding map $V:(M, g) \rightarrow\left(T_{1} M, g_{S}\right)$ is a critical point for the bienergy functional $E_{2}$ (resp. $\left.E_{2}^{v}\right)$, considering only variations through maps defined by unit vector fields. 
The critical point condition for the bienergy functional $E_{2}$ restricted to $\mathfrak{X}^{1}(M)$ has been determined in [7]. More precisely,

Theorem 1.2 Let $(M, g)$ be an m-dimensional Riemannian manifold. Then, $V \in \mathfrak{X}^{1}(M)$ is a biharmonic unit vector field if and only if

$$
\begin{aligned}
\sum_{i=1}^{m}\{ & \left.R\left(e_{i}, \nabla_{e_{i}} S(V)\right) V+\left(\nabla_{e_{i}} R\right)\left(e_{i}, S(V)\right) V+2 R\left(e_{i}, S(V)\right) \nabla_{e_{i}} V\right\} \\
+ & \bar{\Delta}[\bar{\Delta} V-g(\bar{\Delta} V, V) V]-g(\bar{\Delta} V, V) \bar{\Delta} V
\end{aligned}
$$

is collinear to $V$, where $\left\{e_{i}\right\}_{i=1}^{m}$ is a local orthonormal frame field of $(M, g)$.

For the vertical bienergy, $V \in \mathfrak{X}^{1}(M)$ is a biharmonic unit section if and only if

$$
\bar{\Delta}[\bar{\Delta} V-g(\bar{\Delta} V, V) V]-g(\bar{\Delta} V, V) \bar{\Delta} V
$$

is collinear to $V$.

If a unit vector field $V$ of a Riemannian manifold $(M, g)$ defines a harmonic map from $(M, g)$ into $\left(T_{1} M, g_{S}\right)$, i.e. $S(V)=0$ and $\bar{\Delta} V$ is collinear to $V$, then it is automatically a biharmonic unit vector field and biharmonic unit section. Furthermore, if $V$ is a harmonic unit vector field (equivalently, harmonic unit section), i.e. $\bar{\Delta} V$ is collinear to $V$, then it is automatically a biharmonic unit section but, not necessarily a biharmonic unit vector field, unless the manifold is flat.

\section{Examples of biharmonic unit sections which are not harmonic sections}

Example 2.1 On $S U(2)$ equipped with a left-invariant Riemannian metric, take an orthonormal basis $\left\{e_{1}, e_{2}, e_{3}\right\}$ of the Lie algebra $s u(2)$ such that ([9])

$$
\left[e_{2}, e_{3}\right]=\lambda_{1} e_{1}, \quad\left[e_{3}, e_{1}\right]=\lambda_{2} e_{2}, \quad\left[e_{1}, e_{2}\right]=\lambda_{3} e_{3},
$$

where $\lambda_{1}, \lambda_{2}, \lambda_{3}$ are strictly positive constants with $\lambda_{1} \geq \lambda_{2} \geq \lambda_{3}$.

Standard computations (see [7]) and Theorem 1.2 show that a left-invariant unit vector field $V=x_{1} e_{1}+x_{2} e_{2}+x_{3} e_{3}$ is a biharmonic unit section if and only if there exists a real constant $\lambda$ such that

$$
x_{i}\left\{\left(\mu_{j}^{2}+\mu_{k}^{2}\right)^{2}-2\left(\mu_{j}^{2}+\mu_{k}^{2}\right) A-\lambda\right\}=0, \quad \forall i \neq j \neq k \in\{1,2,3\},
$$

where $\mu_{i}=\frac{1}{2}\left(\lambda_{1}+\lambda_{2}+\lambda_{3}\right)-\lambda_{i}(i=1,2,3)$ and $A=\sum_{i \neq j<k}\left(\mu_{j}^{2}+\mu_{k}^{2}\right) x_{i}^{2}$. In combination with [7, Table 2], this yields that:

- If $\lambda_{1}=\lambda_{2}=\lambda_{3}>0$, every left-invariant unit vector field is a biharmonic unit section.

- If $\lambda_{1}=\lambda_{2}>\lambda_{3}, V$ is a non-harmonic biharmonic unit section if and only if $z= \pm \frac{1}{\sqrt{2}}$.

- If $\lambda_{1}>\lambda_{2}=\lambda_{3}, V$ is a non-harmonic biharmonic unit section if and only if $x= \pm \frac{1}{\sqrt{2}}$.

- If $\lambda_{1}>\lambda_{2}>\lambda_{3}$, the only non-harmonic biharmonic unit sections are $\pm \frac{1}{\sqrt{2}} e_{i} \pm \frac{1}{\sqrt{2}} e_{j}$ $(i \neq j)$.

Furthermore, a left-invariant vector field is a biharmonic unit section if and only if is a biharmonic unit vector field. 
Example 2.2 Consider the solvable Lie group $\mathrm{Sol}_{3}$ as $\mathbb{R}^{3}$ equipped with the metric $g_{\text {Sol }}=$ $e^{2 z} d x^{2}+e^{-2 z} d y^{2}+d z^{2}$ and the orthonormal frame field $\left\{e_{1}=e^{-z} \frac{\partial}{\partial x}, e_{2}=e^{z} \frac{\partial}{\partial y}, e_{3}=\frac{\partial}{\partial z}\right\}$. From the connection computed in [10], a left-invariant unit vector field $V=x e_{1}+y e_{2}+z e_{3}$ is a harmonic unit section on $\mathrm{Sol}_{3}$ if and only if

$$
x z^{2}=0, y z^{2}=0, z\left(1-z^{2}\right)=0, x^{2}+y^{2}+z^{2}=1,
$$

i.e. $V= \pm e_{3}$ or $V \in e_{3}^{\perp}$, and $V$ is a (non-harmonic) biharmonic unit section if and only if there exists a constant $\lambda$ such that

$$
x\left(1+2 z^{2}+\lambda\right)=0, y\left(1+2 z^{2}+\lambda\right)=0, z\left(\lambda+4 z^{2}\right)=0, x^{2}+y^{2}+z^{2}=1,
$$

that is $z= \pm \frac{1}{\sqrt{2}}$.

Example 2.3 Let $\left(H^{n}, g\right)$ be the $n$-dimensional hyperbolic space, seen as the $G L(n, \mathbb{R})$ subgroup (cf. [5])

$$
\left(\begin{array}{ccccc}
e^{x_{1}} & 0 & \ldots & 0 & x_{2} \\
0 & e^{x_{1}} & \ldots & 0 & x_{3} \\
\cdot & \cdot & \cdot & \cdot & \cdot \\
\cdot & \cdot & \cdot & \cdot & \cdot \\
0 & \cdot & \cdot & e^{x_{1}} & x_{n} \\
0 & \cdot & \cdot & 0 & 1
\end{array}\right)
$$

This is a solvable Lie group, and its left-invariant vector fields are

$$
X_{1}=c \frac{\partial}{\partial x_{1}}, X_{i}=c e^{x_{1}} \frac{\partial}{\partial x_{i}},(2 \leq i \leq n)
$$

with the metric making this frame orthonormal. The Levi-Civita connection is determined by

$$
\nabla_{X_{i}} X_{i}=c X_{1}, \nabla_{X_{i}} X_{1}=-c X_{i}(2 \leq i \leq n),
$$

and the remaining covariant derivatives vanish.

In [5] it is shown that a left-invariant unit vector field $V$ is always a harmonic unit section when $n=2$ and, for $n>2$, if and only if $V= \pm X_{1}$ or $V \in\left\{X_{1}\right\}^{\perp}$.

Drawing on computations from [5], we obtain that $V=V_{1} X_{1}+\sum_{i=2}^{n} V_{i} X_{i}$ is a biharmonic unit section if and only if there exists a constant $\lambda$ such that

$$
\begin{aligned}
& c^{4}\left\{(n-1)(n-3)-2(n-1)(n-2) V_{1}^{2}\right\} V_{1} \\
& \quad=\lambda V_{1} ;-c^{4}\left\{1+2(n-2) V_{1}^{2}\right\} V_{i}=\lambda V_{i}, 2 \leq i \leq n,
\end{aligned}
$$

and $V$ is a biharmonic unit vector field if and only if there exists a constant $\mu$ such that

$$
\begin{gathered}
c^{4}\left\{(n-1)(n-3)-2(n-1)(n-2) V_{1}^{2}\right\} V_{1}+c^{6}\left\{n+(n-2)(2 n-1) V_{1}^{2}\right\} V_{1}=\mu V_{1}, \\
-c^{4}\left\{1+2(n-2) V_{1}^{2}\right\} V_{i}+c^{6}\left\{2+(n-2)(2 n-1) V_{1}^{2}\right\} V_{i}=\mu V_{i}, \quad 2 \leq i \leq n .
\end{gathered}
$$

Hence, every left-invariant unit vector field on $H^{2}$ is a biharmonic unit section and a biharmonic unit vector field. For $n>2$, a left-invariant unit vector field $V$ is a non-harmonic biharmonic unit section if and only if $V_{1}= \pm \frac{1}{\sqrt{2}}$; left-invariant biharmonic unit vector fields are $V= \pm X_{1}, V \in X_{1}^{\perp}$ or satisfy $V_{1}= \pm \sqrt{\frac{c^{2}+n-2}{2(n-2)}},\left(c^{2}<n-2\right)$. 
Remark 2.1 The notions of "harmonic unit section" and "harmonic unit vector fields" are equivalent. On the other hand, there exist left-invariant unit vector fields of $H^{n}$ which are biharmonic unit sections (resp. biharmonic unit vector fields) which are not biharmonic unit vector fields (resp. biharmonic unit sections).

Remark 2.2 Example 2.3 completes and corrects the statement of [6, Theorem 3.1].

\section{Conformal change of the metric and the tension field of a vector field}

In this section, we derive the critical points of the bienergy functional (resp. vertical bienergy functional) for unit vector fields after a conformal change of the metric on the base manifold. We have

Theorem 3.1 Let $\left(M^{n}, g\right)$ be a Riemannian manifold, $V$ a unit vector field on $M$ and $u \in$ $C^{\infty}(M)$. We consider the conformal change of metric $\widetilde{g}=e^{2 u} g$ and $\widetilde{V}=e^{-u} V$. Then,

$$
\begin{aligned}
\tilde{\bar{\Delta}} \tilde{V}-\tilde{g}(\tilde{\bar{\Delta}} \widetilde{V}, \tilde{V}) \tilde{V}= & e^{-3 u}\left\{\bar{\Delta} V-g(\bar{\Delta} V, V) V+(2-n) \nabla_{\operatorname{grad} u} V\right. \\
& -2 \sum_{i=1}^{n-1} g\left(\operatorname{grad} u, \nabla_{E_{i}} V\right) E_{i} \\
& \left.+[2 \operatorname{div} V+(n-2) V(u)] \sum_{i=1}^{n-1} E_{i}(u) E_{i}\right\}, \\
\widetilde{\nabla} \widetilde{V} \widetilde{V}-(\widetilde{\operatorname{div}} \tilde{V}) \tilde{V}= & e^{-2 u}\left\{\nabla_{V} V-(\operatorname{div} V) V+(2-n) V(u) V-\operatorname{grad} u\right\},
\end{aligned}
$$

where $\left\{E_{1}, E_{2}, \ldots, E_{n-1}, E_{n}=V\right\}$ is an orthonormal frame field of $(M, g)$.

Proof Consider an arbitrary orthonormal frame field $\left\{E_{1}, E_{2}, \ldots, E_{n}\right\}$ of $(M, g)$ and set $\widetilde{E}_{i}=e^{-u} E_{i}(1 \leq i \leq n)$ an orthonormal frame field of $(M, \widetilde{g})$. By standard computations (cf. [3]), we have

$$
\widetilde{\nabla}_{\widetilde{E}_{i}} \widetilde{V}=e^{-2 u}\left\{\nabla_{E_{i}} V+V(u) E_{i}-g\left(V, E_{i}\right) \operatorname{grad} u\right\} .
$$

Using repeatedly (3.3), we get (summing on repeated indices)

$$
\begin{aligned}
\widetilde{\nabla}_{\widetilde{E}_{i}} \widetilde{\nabla}_{\widetilde{E}_{i}} \widetilde{V}= & e^{-3 u}\left\{-\nabla_{\operatorname{grad} u} V+\nabla_{E_{i}} \nabla_{E_{i}} V-2 \operatorname{div} V \operatorname{grad} u+\left(\nabla_{E_{i}} V\right)(u) E_{i}+E_{i}[V(u)] E_{i}\right. \\
& \left.+V(u) \nabla_{E_{i}} E_{i}+(2-n) V(u) E_{i}(u) E_{i}-g\left(V, \nabla_{E_{i}} E_{i}\right) \operatorname{grad} u-\nabla_{V} \operatorname{grad} u-\|\operatorname{grad} u\|^{2} V\right\}, \\
\widetilde{\nabla}_{\widetilde{E}_{i}} \widetilde{E}_{i}= & e^{-2 u}\left\{(1-n) \operatorname{grad} u+\nabla_{E_{i}} E_{i}\right\}, \\
\widetilde{\nabla}_{\widetilde{\nabla}_{\widetilde{E}_{i}} \widetilde{E}_{i}} \widetilde{V}= & e^{-3 u}\left\{(1-n) \nabla_{\operatorname{grad} u} V+\nabla_{\nabla_{E_{i}}} E_{i} V+V(u) \nabla_{E_{i}} E_{i}-g\left(\nabla_{E_{i}} E_{i}, V\right) \operatorname{grad} u\right\} .
\end{aligned}
$$

As a consequence,

$$
\begin{aligned}
\widetilde{\bar{\Delta}} \widetilde{V} & =e^{-3 u}\left\{\bar{\Delta} V+(2-n) \nabla_{\operatorname{grad}} V+\nabla_{V} \operatorname{grad} u+2 \operatorname{div} V \operatorname{grad} u-\left(\nabla_{E_{i}} V\right)(u) E_{i}\right. \\
& \left.+(n-2) V(u) \operatorname{grad} u-E_{i}[V(u)] E_{i}+\|\operatorname{grad} u\|^{2} V\right\}, \\
\widetilde{g}(\widetilde{\bar{\Delta}} \widetilde{V}, \widetilde{V})= & e^{-2 u}\left\{g(\bar{\Delta} V, V)+g\left(\nabla_{V} \operatorname{grad} u, V\right)+2 \operatorname{div} V V(u)-\left(\nabla_{V} V\right)(u)\right. \\
& \left.+(n-2) V(u)^{2}-V V(u)+\|\operatorname{grad} u\|^{2}\right\} .
\end{aligned}
$$


Then take $E_{n}=V$ to obtain (3.1), while (3.2) follows from (3.3).

Corollary 3.2 Let $\left(M^{2}, g\right)$ be a two-dimensional Riemannian manifold, $V$ a unit vector field on $M$ and $u \in C^{\infty}(M)$. Consider the conformal change $\widetilde{g}=e^{2 u} g$ and $\widetilde{V}=e^{-u} V$. Then,

$$
\tilde{\bar{\Delta}} \widetilde{V}-\tilde{g}(\tilde{\bar{\Delta}} \widetilde{V}, \widetilde{V}) \widetilde{V}=e^{-3 u}\{\bar{\Delta} V-g(\bar{\Delta} V, V) V\} .
$$

So $V$ is a harmonic unit section on $\left(M^{2}, g\right)$ if and only if $\tilde{V}$ is a harmonic unit section on $\left(M^{2}, \widetilde{g}\right)$.

Remark 3.1 Beware that the above property is of a different nature from the conformal invariance of harmonic maps on surfaces.

Theorem 3.3 Let $\left(M^{2}, g\right)$ be a two-dimensional compact Riemannian manifold, $V$ a unit vectorfield on $M$ and $u \in C^{\infty}(M)$. Consider the conformal change $\widetilde{g}=e^{2 u} g$ and $\widetilde{V}=e^{-u} V$. Then $V$ is a biharmonic unit section if and only if

$$
\widetilde{\bar{\Delta}}\left(e^{2 u}(\tilde{\bar{\Delta}} \widetilde{V}-\widetilde{g}(\tilde{\bar{\Delta}} \widetilde{V}, \widetilde{V}) \widetilde{V})\right)-e^{2 u} \widetilde{g}(\tilde{\bar{\Delta}} \widetilde{V}, \widetilde{V}) \widetilde{\bar{\Delta}} \widetilde{V}
$$

is collinear to $\widetilde{V}$.

Proof We adopt the terminology of [7] and, due to Corollary 3.2, consider the function

$$
E_{2}^{v}(t)=\frac{1}{2} \int_{M} e^{2 u}\left\{\widetilde{g}\left(\tilde{\bar{\Delta}} \widetilde{V}_{t}, \widetilde{\bar{\Delta}} \widetilde{V}_{t}\right)-\widetilde{g}\left(\widetilde{\bar{\Delta}} \widetilde{V}_{t}, \widetilde{V}_{t}\right)^{2}\right\} v \widetilde{g} .
$$

For the sake of convenience, we omit the tilde. From the proof of [7, Theorem 3.2], the derivative of $E_{2}^{v}$ becomes

$$
\begin{aligned}
\frac{\mathrm{d}}{\mathrm{d} t} E_{2}^{v}(t)= & \int_{M} e^{2 u} \Delta\left[g\left(\nabla_{\partial_{t}} V_{t}, \bar{\Delta} V_{t}\right)\right] v_{g}+2 \int_{M} e^{2 u} \operatorname{div} \theta_{t} v_{g}+\int_{M} e^{2 u} g\left(\bar{\Delta} \bar{\Delta} V_{t}, \nabla_{\partial_{t}} V_{t}\right) v_{g} \\
& -2 \int_{M} e^{2 u}\left(\operatorname{div} \omega_{t}\right) g\left(\bar{\Delta} V_{t}, V_{t}\right) v_{g}-2 \int_{M} e^{2 u} g\left(\bar{\Delta} V_{t}, V_{t}\right) g\left(\bar{\Delta} V_{t}, \nabla_{\partial_{t}} V_{t}\right) v_{g}
\end{aligned}
$$

where $\theta_{t}(\cdot)=g\left(\nabla_{\partial_{t}} V_{t}, \nabla \cdot \bar{\Delta} V_{t}\right)$ and $\omega_{t}(\cdot)=g\left(\nabla_{\partial_{t}} V_{t}, \nabla \cdot V_{t}\right)(t \in I)$ and further simplifies at $t=0$ into

$$
\left.\frac{\mathrm{d}}{\mathrm{d} t}\right|_{t=0} E_{2}^{v}(t)=\int_{M} \tilde{g}\left(X, \tilde{\bar{\Delta}}\left(e^{2 u}(\tilde{\bar{\Delta}} \widetilde{V}-\widetilde{g}(\tilde{\bar{\Delta}} \widetilde{V}, \widetilde{V}) \widetilde{V})\right)-e^{2 u} \widetilde{g}(\tilde{\bar{\Delta}} \widetilde{V}, \widetilde{V}) \widetilde{\bar{\Delta}} \widetilde{V}\right) v_{\tilde{g}},
$$

with $X=\left.\nabla_{\partial_{t}} V_{t}\right|_{t=0}$.

For a two-dimensional manifold $(M, g)$ with Gaussian curvature $k_{g}$ and $V$ a unit vector field on $M$, we have $S(V)=k_{g}\left(\nabla_{V} V-\operatorname{div}(V) V\right)$. By (3.2), with $n=2$, we obtain

$$
\begin{aligned}
E_{2}(V)= & \frac{1}{2} \int_{M}\left\{e^{2 u}\|\widetilde{\bar{\Delta}} \widetilde{V}-\widetilde{g}(\widetilde{\bar{\Delta}} \widetilde{V}, \widetilde{V}) \widetilde{V}\|_{\widetilde{g}}^{2}+k_{g}^{2}\left[\left\|\widetilde{\nabla_{V}} \widetilde{V}-\widetilde{\operatorname{div}}(\widetilde{V}) \widetilde{V}\right\|_{\widetilde{g}}^{2}+\|\widetilde{\operatorname{grad}} u\|_{\widetilde{g}}^{2}\right.\right. \\
& +2 \widetilde{g}(\widetilde{\nabla} \widetilde{V} \widetilde{V}-\widetilde{\operatorname{div}}(\widetilde{V}) \widetilde{V}, \widetilde{\operatorname{grad} u})]\} v \widetilde{g} .
\end{aligned}
$$

In the sequel, we derive a condition on $\widetilde{V}$ for $V$ to be a biharmonic unit vector field. It is enough to deal with the derivative of horizontal part of the integral since the vertical part was treated in Theorem 3.3. 
Theorem 3.4 Let $\left(M^{2}, g\right)$ be a two-dimensional compact Riemannian manifold, $V$ a unit vector field on $M$ and $u \in C^{\infty}(M)$. We consider the conformal change of metric $\widetilde{g}=e^{2 u} g$ such that $(M, \widetilde{g})$ is flat and $\widetilde{V}=e^{-u} V$. Then, $V$ is a biharmonic unit vector field if and only if,

$$
\begin{aligned}
& \tilde{\bar{\Delta}}\left(e^{2 u}(\tilde{\bar{\Delta}} \tilde{V}-\tilde{g}(\tilde{\bar{\Delta}} \tilde{V}, \tilde{V}) \tilde{V})\right)-e^{2 u} \tilde{g}(\tilde{\bar{\Delta}} \tilde{V}, \tilde{V}) \tilde{\bar{\Delta}} \tilde{V}+[\tilde{V}, J \tilde{V}] k_{g}^{2} J \tilde{V} \\
& \quad+k_{g}^{2}(\tilde{\bar{\Delta}} \tilde{V}-\tilde{g}(\tilde{\bar{\Delta}} \tilde{V}, \tilde{V}) \tilde{V})+\tilde{g}\left(\widetilde{\operatorname{grad}} u, \widetilde{\operatorname{grad}} k_{g}^{2}\right) J \tilde{V}
\end{aligned}
$$

is collinear to $\widetilde{V}$, where $J$ is the natural complex structure on $\left(M^{2}, g\right)$.

Proof Using the orthonormal frame field $\left\{\widetilde{E}_{1}=J \widetilde{V}, \widetilde{V}\right\}$ of $(M, \widetilde{g})$, we have

$$
\widetilde{\bar{\Delta}} V=\widetilde{\nabla}_{\widetilde{\nabla}_{\widetilde{E}_{1}}} \widetilde{E}_{1} \widetilde{V}-\widetilde{\nabla}_{\widetilde{E}_{1}} \widetilde{\nabla}_{\widetilde{E}_{1}} \widetilde{V}+\widetilde{\nabla}_{\widetilde{\nabla}_{\tilde{V}}} \widetilde{V} \widetilde{V}-\widetilde{\nabla}_{\widetilde{V}} \widetilde{\nabla}_{\widetilde{V}} \widetilde{V}
$$

and

$$
\widetilde{g}(\widetilde{\bar{\Delta}} \widetilde{V}-\widetilde{g}(\widetilde{\bar{\Delta}} \widetilde{V}, \widetilde{V}) \widetilde{V}, J \widetilde{V})=\widetilde{V}(\widetilde{\operatorname{div}} J \widetilde{V})-J \widetilde{V}(\widetilde{\operatorname{div}} \widetilde{V}) .
$$

We consider the horizontal part of the bienergy functional of $\tilde{V}$ :

$F(t)=\frac{1}{2} \int_{M}\left\{k_{g}^{2}\left\|\widetilde{\nabla}_{\widetilde{V}_{t}} \widetilde{V}_{t}-\left(\widetilde{\operatorname{div}} \widetilde{V}_{t}\right) \widetilde{V}_{t}\right\|_{\widetilde{g}}^{2}+2 k_{g}^{2} \widetilde{g}\left(\widetilde{\nabla}_{\widetilde{V}_{t}} \widetilde{V}_{t}-\left(\widetilde{\operatorname{div}} \widetilde{V}_{t}\right) \widetilde{V}_{t}, \widetilde{\operatorname{grad}} u\right)+k_{g}^{2}\|\widetilde{\operatorname{grad}} u\|_{\widetilde{g}}^{2}\right\} v \widetilde{g}$.

As previously, we momentarily omit the tilde for readability. Assuming that $(M, \tilde{g})$ is flat, we have for the first term of $\frac{\mathrm{d} F}{\mathrm{~d} t}$

$$
\begin{aligned}
\frac{\mathrm{d}}{\mathrm{d} t} & \frac{1}{2} \int_{M} k_{g}^{2}\left(g\left(\nabla_{V_{t}} V_{t}, \nabla_{V_{t}} V_{t}\right)+\left(\operatorname{div} V_{t}\right)^{2}\right) v_{g} \\
& =\int_{M}\left\{k_{g}^{2} g\left(\nabla_{\partial_{t}} \nabla_{V_{t}} V_{t}, \nabla_{V_{t}} V_{t}\right)+k_{g}^{2}\left(\operatorname{div} V_{t}\right) \frac{\mathrm{d}}{\mathrm{d} t}\left[\operatorname{div} V_{t}\right]\right\} v_{g} \\
& =\int_{M}\left(k_{g}^{2} g\left(\nabla_{V_{t}} \nabla_{\partial_{t}} V_{t}+\nabla_{\nabla_{\partial_{t}} V_{t}} V_{t}, \nabla_{V_{t}} V_{t}\right)+k_{g}^{2}\left(\operatorname{div} V_{t}\right) g\left(\nabla_{e_{i}} \nabla_{\partial_{t}} V_{t}, e_{i}\right)\right) v_{g} \\
& =\int_{M}\left(k_{g}^{2} g\left(\nabla_{V_{t}} \nabla_{\partial_{t}} V_{t}+\nabla_{\nabla_{\partial_{t}} V_{t}} V_{t}, \nabla_{V_{t}} V_{t}\right)-g\left(\nabla_{\partial_{t}} V_{t}, \operatorname{grad}\left(k_{g}^{2} \operatorname{div} V_{t}\right)\right)\right) v_{g},
\end{aligned}
$$

where $\eta_{t}(\cdot)=g\left(\nabla_{\partial_{t}} V_{t}, \cdot\right)$. We set $X=\left.\nabla_{\partial_{t}} V_{t}\right|_{t=0}$, so

$$
\begin{aligned}
& \frac{1}{2}\left.\frac{\mathrm{d}}{\mathrm{d} t}\right|_{t=0} \int_{M} k_{g}^{2}\left(g\left(\nabla_{V_{t}} V_{t}, \nabla_{V_{t}} V_{t}\right)+\left(\operatorname{div} V_{t}\right)^{2}\right) v_{g} \\
& \quad=\int_{M} k_{g}^{2} g\left(\nabla_{V} X+\nabla_{X} V, \nabla_{V} V\right)-g\left(X, \operatorname{grad}\left[k_{g}^{2} \operatorname{div} V\right]\right) v_{g} .
\end{aligned}
$$

Since $X$ is perpendicular to $V, X=g(X, J V) J V=f J V$ and, with $a=-\operatorname{div} J V$ and $b=\operatorname{div} V$, we have

$k_{g}^{2} g\left(\nabla_{V} X+\nabla_{X} V, \nabla_{V} V\right)=k_{g}^{2}(a V(f)+a b f)=\operatorname{div}\left(a k_{g}^{2} f V\right)+g(X, J V) V\left(\operatorname{div}(J V) k_{g}^{2}\right)$,

so

$$
\begin{aligned}
& V\left(\operatorname{div}(J V) k_{g}^{2}\right) J(V)-g\left(\operatorname{grad}\left[k_{g}^{2} \operatorname{div} V\right], J V\right) J V \\
& \quad=[V, J V]\left(k_{g}^{2}\right) J V+k_{g}^{2}(\bar{\Delta} V-g(\bar{\Delta} V, V) V, J V) J V .
\end{aligned}
$$


For the other term of $\frac{\mathrm{d} F}{\mathrm{~d} t}$, we have

$$
\begin{aligned}
\frac{\mathrm{d}}{\mathrm{d} t} & \int_{M} k_{g}^{2} g\left(\nabla_{V_{t}} V_{t}-\left(\operatorname{div} V_{t}\right) V_{t}, \operatorname{grad} u\right) v_{g} \\
= & \int_{M} k_{g}^{2} g\left(\nabla_{\partial_{t}} \nabla_{V_{t}} V_{t}-\left[\frac{\mathrm{d}}{\mathrm{d} t} \operatorname{div} V_{t}\right] V_{t}-\operatorname{div} V_{t} \nabla_{\partial_{t}} V_{t}, \operatorname{grad} u\right) v_{g} \\
= & \int_{M} k_{g}^{2} g\left(\nabla_{V_{t}} \nabla_{\partial_{t}} V_{t}+\nabla_{\nabla_{\partial_{t}}} V_{t} V_{t}, \operatorname{grad} u\right) v_{g}-\int_{M} \operatorname{div}\left(k_{g}^{2} V_{t}(u) \eta_{t}\right) v_{g} \\
& +\int_{M} \eta_{t}\left(\operatorname{grad}\left[k_{g}^{2} V_{t}(u)\right]\right) v_{g}-\int_{M} k_{g}^{2} g\left(\nabla_{\partial_{t}} V_{t}, \operatorname{div} V_{t} \operatorname{grad} u\right) v_{g} .
\end{aligned}
$$

As a consequence,

$$
\begin{aligned}
& \left.\frac{\mathrm{d}}{\mathrm{d} t}\right|_{t=0} \int_{M} k_{g}^{2} g\left(\nabla_{V_{t}} V_{t}-\left(\operatorname{div} V_{t}\right) V_{t}, \operatorname{grad} u\right) v_{g}=\int_{M} k_{g}^{2} g\left(\nabla_{V} X+\nabla_{X} V, \operatorname{grad} u\right) v_{g} \\
& \quad+\int_{M} g\left(X, \operatorname{grad}\left[k_{g}^{2} V(u)\right]-k_{g}^{2}(\operatorname{div} V) \operatorname{grad} u\right) v_{g},
\end{aligned}
$$

where $X=f J V$. We have

$$
\begin{aligned}
k_{g}^{2} g\left(\nabla_{V} X+\nabla_{X} V, \operatorname{grad} u\right)= & \operatorname{div}\left(k_{g}^{2} J V(u) f V\right)-g(J V, X) V\left(k_{g}^{2} J V(u)\right) \\
& +k_{g}^{2} g(J V, X) V(u) \operatorname{div} J V,
\end{aligned}
$$

and

$$
\begin{aligned}
& -V\left(k_{g}^{2} J V(u)\right)+k_{g}^{2}[\operatorname{div}(J V)] V(u)+J V\left(k_{g}^{2} V(u)\right)-k_{g}^{2}(\operatorname{div} V) J V(u) \\
& =-V\left(k_{g}^{2}\right) E_{1}(u)+E_{1}\left(k_{g}^{2}\right) V(u)=g\left(J \operatorname{grad} u, \operatorname{grad} k_{g}^{2}\right) .
\end{aligned}
$$

This yields (3.5).

\section{Biharmonic unit sections and biharmonic unit vector fields on 2-torus}

Let $d_{1}, d_{2} \in \mathbb{R}^{2}$ be two linearly independent vectors and $\Gamma \subset \mathbb{R}^{2}$ the lattice given by $\Gamma=\left\{m d_{1}+n d_{2}: m, n \in \mathbb{Z}\right\}$. Consider the 2-torus $T^{2}=\mathbb{R}^{2} / \Gamma$ and the natural projection $\pi: \mathbb{R}^{2} \rightarrow T^{2}$. Then $\mathbb{R}^{2}$ is the universal covering space of $T^{2}$. Let $J$ be the complex structure on $T^{2}$ induced by the fixed orientation. Take an arbitrary Riemannian metric $g$ on $T^{2}, k_{g}$ its Gaussian curvature and $\{S, W\}$ an orthonormal frame field such that $J S=W$.

Definition 4.1 $[3,11]$ Let $(m, n) \in \mathbb{Z}^{2}$. We set

$$
\operatorname{Per}(m, n)=\left\{\alpha \in C^{\infty}\left(\mathbb{R}^{2}\right): \alpha\left(\xi+d_{1}\right)-\alpha(\xi)=2 m \pi, \alpha\left(\xi+d_{2}\right)-\alpha(\xi)=2 n \pi, \forall \xi \in \mathbb{R}^{2}\right\} .
$$

An element $\alpha \in \operatorname{Per}(m, n)$ is referred to as an $(m, n)$-semiperiodic function, and we set $\mathcal{W}=\bigcup_{(m, n) \in \mathbb{Z}^{2}} \operatorname{Per}(m, n)$.

Let $V$ be a unit vector field on $\left(T^{2}, g\right)$. A function $\alpha: \mathbb{R}^{2} \rightarrow \mathbb{R}$ is called an $(S, W)$-angle function for $V$ if, on the whole of $\mathbb{R}^{2}$,

$$
V \circ \pi=(\cos \alpha) S \circ \pi+(\sin \alpha) W \circ \pi .
$$

Theorem 4.2 Let $\left(T^{2}, g\right)$ be a two-dimensional Riemannian torus. Then: 
(i) A unit vector field $V$ on $\left(T^{2}, g\right)$ is a biharmonic unit section if and only if it is a harmonic unit section.

(ii) There exist biharmonic unit vector fields on $\left(T^{2}, g\right)$.

Proof (i) Let $u \in C^{\infty}\left(T^{2}\right)$ and $\widetilde{g}=e^{2 u} g$ be the metric on $T^{2}$ conformal to $g$ such that $\left(T^{2}, \widetilde{g}\right)$ is flat. Take $\left\{V_{1}, V_{2}\right\}$ a parallel $\widetilde{g}$-orthonormal frame field and $\widetilde{V}=e^{-u} V$. Then $\widetilde{V}=\cos \alpha V_{1}+\sin \alpha V_{2}$ for a smooth function $\alpha: T^{2} \rightarrow \mathbb{R}$. We compute

$$
\begin{aligned}
& \widetilde{\bar{\Delta}} \widetilde{V}=\left[-\sin \alpha \widetilde{\Delta} \alpha+(\cos \alpha)\|\widetilde{\operatorname{grad}} \alpha\|^{2}\right] V_{1}+\left[(\cos \alpha) \widetilde{\Delta} \alpha+(\sin \alpha)\|\widetilde{\operatorname{grad}} \alpha\|^{2}\right] V_{2} ; \\
& \widetilde{g}(\widetilde{\bar{\Delta}} \widetilde{V}, \widetilde{V})=\|\widetilde{\operatorname{grad}} \alpha\|^{2}, \widetilde{g}(\widetilde{\bar{\Delta}} \widetilde{V}, J \widetilde{V})=\widetilde{\Delta} \alpha, \widetilde{g}(\widetilde{\bar{\Delta}}(J \widetilde{V}), J \widetilde{V})=\|\widetilde{\operatorname{grad}} \alpha\|^{2} ; \\
& \widetilde{\bar{\Delta}} \widetilde{V}-\widetilde{g}(\tilde{\bar{\Delta}} \widetilde{V}, \widetilde{V}) \widetilde{V}=-\sin \alpha \widetilde{\Delta} \alpha V_{1}+(\cos \alpha) \widetilde{\Delta} \alpha V_{2}=\widetilde{\Delta} \alpha J(\widetilde{V}) ; \\
& \left.\widetilde{\bar{\Delta}}\left(e^{2 u}(\widetilde{\bar{\Delta}} \widetilde{V}-\widetilde{g}(\widetilde{\bar{\Delta}} \widetilde{V}, \widetilde{V}) \widetilde{V})\right)=\widetilde{\Delta}\left(e^{2 u} \widetilde{\Delta} \alpha\right) J \widetilde{V}+e^{2 u}(\widetilde{\Delta} \alpha) \widetilde{\bar{\Delta}}(J \widetilde{V})-\widetilde{\operatorname{grad}\left[e^{2 u}\right.} \widetilde{\Delta} \alpha\right] J \widetilde{V},
\end{aligned}
$$

where $J \widetilde{V}=-\sin \alpha V_{1}+\cos \alpha V_{2}$. Using (4.1) and (3.4), we have $\widetilde{\Delta}\left(e^{2 u} \widetilde{\Delta} \alpha\right)=0$, so $\alpha$ is a constant function on $T^{2}$ and $\widetilde{V}$ is a harmonic unit section on $\left(T^{2}, \widetilde{g}\right)$. By Corollary $3.2, V$ is also a harmonic unit section on $\left(T^{2}, g\right)$.

(ii) Since $V_{1}, V_{2}$ are parallel vector fields on $\left(T^{2}, \widetilde{g}\right)$, we have

$$
\begin{gathered}
\widetilde{\nabla}_{\widetilde{V}} J \widetilde{V}=\left\{-\cos ^{2} \alpha V_{1}(\alpha)-\sin \alpha \cos \alpha V_{2}(\alpha)\right\} V_{1}+\left\{-\sin \alpha \cos \alpha V_{1}(\alpha)-\sin ^{2} \alpha V_{2}(\alpha)\right\} V_{2}, \\
\widetilde{\nabla}_{J \widetilde{V}} \widetilde{V}=\left\{\sin ^{2} \alpha V_{1}(\alpha)-\sin \alpha \cos \alpha V_{2}(\alpha)\right\} V_{1}+\left\{-\sin \alpha \cos \alpha V_{1}(\alpha)+\cos ^{2} \alpha V_{2}(\alpha)\right\} V_{2}, \\
{[\widetilde{V}, J \widetilde{V}]=\widetilde{\nabla}_{\widetilde{V}} J \widetilde{V}-\widetilde{\nabla}_{J \widetilde{V}} \widetilde{V}=-\widetilde{\operatorname{grad}} \alpha .}
\end{gathered}
$$

Taking the inner product of (3.5) with $J \widetilde{V}$, we obtain that $V$ is a biharmonic unit vector field on $\left(T^{2}, g\right)$ if and only if

$$
\widetilde{\Delta}\left(e^{2 u} \widetilde{\Delta} \alpha\right)-\widetilde{\operatorname{div}}\left(k_{g}^{2} \widetilde{\operatorname{grad}} \alpha\right)=-\widetilde{\operatorname{div}}\left(k_{g}^{2} \widetilde{\operatorname{grad}} u\right) .
$$

The left-hand side is an elliptic, self-adjoint operator $P$ whose kernel is reduced to constant functions, since $P h=0$ implies

$$
\int_{T^{2}} e^{2 u}(\widetilde{\Delta} h)^{2} v_{\widetilde{g}}=-\int_{T^{2}} k_{g}^{2}\|\widetilde{\operatorname{grad}} h\|^{2} v_{\widetilde{g}} .
$$

Therefore, Eq. (4.2) admits a solution if its right-hand term lies in (ker $P)^{\perp}$ (cf. [2]), which, being a divergence, is clearly the case.

Remark 4.1 In [11], Wiegmink showed that, unlike biharmonic unit vector fields, critical points of the total bending on a two-dimensional torus are conformally invariant.

Recall that [11, Lemma 3] shows that, for a given global frame field $\{S, W\}$, homotopy classes of unit vector fields on $T^{2}$ are classified by their semi-periodicity class in $\mathbb{Z}^{2}$.

The aim of this section is to compute critical points of the bienergy functional of unit vector fields on $\left(T^{2}, g\right)$ defined on the set $\mathcal{W}$ of all angle functions. Fix an orthonormal frame field $\{S, W\}$ of $\left(T^{2}, g\right)$, then

$$
\nabla_{S} S=\alpha W, \nabla_{S} W=-\alpha S, \nabla_{W} S=b W, \nabla_{W} W=-b S, \operatorname{div}(Z)=S(\alpha)+W(b),
$$

where $Z=\alpha S+b W \in \mathfrak{X}\left(T^{2}\right)$. We consider the 1-forms $\Theta_{S}, \Theta_{W} \in \Omega^{1}\left(T^{2}\right)$ and the volume form $\omega_{T}=\Theta_{S} \wedge \Theta_{W}$. Let $V$ be a unit vector field on $\left(T^{2}, g\right)$ and $\theta \in \mathcal{W}$ its angle function, then, 


$$
\widehat{V} \circ \pi=(\cos \theta) S \circ \pi+(\sin \theta) W \circ \pi,
$$

where $\pi: \mathbb{R}^{2} \rightarrow T^{2}$ is the projection. We set $\widehat{g}=\pi^{*} g$. We denote by $\widehat{S}, \widehat{W}, \widehat{V}$ vector fields on $\mathbb{R}^{2}$ which are $\pi$-related to $S, W, V$, i.e. $d \pi_{p}(\widehat{S}(p))=S(\pi(p))$ for all $p \in \mathbb{R}^{2}$. The hat symbol indicates dependence on the pullback metric $\widehat{g}$. We have

Lemma 4.3 Let $V$ be a unit vector field on $\left(T^{2}, g\right)$ and $\theta$ its angle function. Then,

$$
\begin{aligned}
& \widehat{\bar{\Delta}} \widehat{V}-\widehat{g}(\widehat{\bar{\Delta}} \widehat{V}, \widehat{V}) \widehat{V}=\{-(\sin \theta) \widehat{\Delta} \theta+(\sin \theta)[\widehat{W}(b \circ \pi)+\widehat{S}(\alpha \circ \pi)]\} \widehat{S} \\
&+\{(\cos \theta) \widehat{\Delta} \theta-(\cos \theta)[\widehat{S}(\alpha \circ \pi)+\widehat{W}(b \circ \pi)]\} \widehat{W}, \\
& \widehat{\nabla} \widehat{V} \widehat{V}-(\widehat{\operatorname{div}} \widehat{V}) \widehat{V}=\{-\widehat{W}(\theta)-b \circ \pi\} \widehat{S}+\{\alpha \circ \pi+\widehat{S}(\theta)\} \widehat{W} .
\end{aligned}
$$

Proof By (4.3), we get

$$
\begin{aligned}
\widehat{\bar{\Delta}} \widehat{S} & =\left\{(\alpha \circ \pi)^{2}+(b \circ \pi)^{2}\right\} \widehat{S}+\{-\widehat{S}(\alpha \circ \pi)-\widehat{W}(b \circ \pi)\} \widehat{W}, \\
\widehat{\bar{\Delta}} \widehat{W} & =\{\widehat{W}(b \circ \pi)+\widehat{S}(\alpha \circ \pi)\} \widehat{S}+\left\{(\alpha \circ \pi)^{2}+(b \circ \pi)^{2}\right\} \widehat{W}, \\
\widehat{\Delta}(\cos \theta) & =(\cos \theta)\|\widehat{\operatorname{grad}} \theta\|^{2}-(\sin \theta) \widehat{\Delta} \theta, \\
\widehat{\Delta}(\sin \theta) & =(\sin \theta)\|\widehat{\operatorname{grad}} \theta\|^{2}+(\cos \theta) \widehat{\Delta} \theta .
\end{aligned}
$$

Combining (4.3), (4.5) and $\widehat{V}=(\cos \theta) \widehat{S}+(\sin \theta) \widehat{W}$, we compute

$$
\begin{aligned}
\widehat{\bar{\Delta}} \widehat{V}= & \left\{(\cos \theta)\|\widehat{\operatorname{grad}} \theta\|^{2}-(\sin \theta) \widehat{\Delta} \theta+(\cos \theta)\left[(\alpha \circ \pi)^{2}+(b \circ \pi)^{2}\right]+(\sin \theta)[\widehat{W}(b \circ \pi)\right. \\
& +\widehat{S}(\alpha \circ \pi)]+2 \widehat{S}(\theta)(\alpha \circ \pi) \cos \theta+2 \widehat{W}(\theta)(b \circ \pi) \cos \theta\} \widehat{S} \\
& +\left\{(\sin \theta)\|\widehat{\operatorname{grad}} \theta\|^{2}+(\cos \theta) \widehat{\Delta} \theta+(\sin \theta)\left[(\alpha \circ \pi)^{2}+(b \circ \pi)^{2}\right]\right. \\
& -(\cos \theta)[\widehat{S}(\alpha \circ \pi)+\widehat{W}(b \circ \pi)]+2 \sin \theta(\alpha \circ \pi) \widehat{S}(\theta)+2 \sin \theta(b \circ \pi) \widehat{W}(\theta)\} \widehat{W},
\end{aligned}
$$

$\widehat{g}(\widehat{\bar{\Delta}} \widehat{V}, \widehat{V})=\|\widehat{\operatorname{grad}} \theta\|^{2}+(\alpha \circ \pi)^{2}+(b \circ \pi)^{2}+2 \widehat{S}(\theta)(\alpha \circ \pi)+2 \widehat{W}(\theta)(b \circ \pi)$,

$$
\begin{aligned}
\widehat{\nabla}_{\widehat{V}} \widehat{V}= & {\left[-\sin \theta \cos \theta \widehat{S}(\theta)-\sin \theta \cos \theta(\alpha \circ \pi)-\left(\sin ^{2} \theta\right) \widehat{W}(\theta)-(b \circ \pi) \sin ^{2} \theta\right] \widehat{S} } \\
& +\left[\cos ^{2} \theta(\alpha \circ \pi)+\left(\cos ^{2} \theta\right) \widehat{S}(\theta)+\sin \theta \cos \theta(b \circ \pi)+\sin \theta \cos \theta \widehat{W}(\theta)\right] \widehat{W},
\end{aligned}
$$

$\widehat{\operatorname{div}} \widehat{V}=\cos \theta(b \circ \pi)-(\sin \theta) \widehat{S}(\theta)-\sin \theta(\alpha \circ \pi)+(\cos \theta) \widehat{W}(\theta)$,

to immediately obtain (4.4).

Let $Q=\left\{s d_{1}+t d_{2} \in \mathbb{R}^{2}:(s, t) \in[0,1]^{2}\right\}$. Then $\pi(Q)=T^{2}$ and $\pi: Q \backslash \partial Q \rightarrow T^{2}$ is injective. By Lemma 4.3, we have

$$
\begin{aligned}
E_{2}(V) & =\frac{1}{2} \int_{T^{2}}\left\{\|\bar{\Delta} V-g(\bar{\Delta} V, V) V\|_{g}^{2}+k_{g}^{2}\left\|\nabla_{V} V-(\operatorname{div} V) V\right\|_{g}^{2}\right\} v_{g} \\
& =\int_{Q}\left\{(\widehat{\Delta} \theta-\widehat{\operatorname{div}} \widehat{Z})^{2}+\left(k_{g} \circ \pi\right)^{2}\|\widehat{\operatorname{grad} \theta}+\widehat{Z}\|_{\widehat{g}}^{2}\right\} \pi^{*}\left(\omega_{T}\right) \\
& =G(\theta),
\end{aligned}
$$

where $v_{g}=2 \omega_{T}$ and $\widehat{Z}=(\alpha \circ \pi) \widehat{S}+(b \circ \pi) \widehat{W}$ ([11]). Next, we characterize critical points of the functional $G: \mathcal{W} \rightarrow[0,+\infty)$ on the set $\mathcal{W}$. 
Theorem 4.4 Let $\left(T^{2}, g\right)$ be a 2-torus, $k_{g}$ its Gaussian curvature and $\{S, W\}$ an orthonormal frame field of $\left(T^{2}, g\right)$. Then, (i) $V \in \mathfrak{X}^{1}\left(T^{2}\right)$ is a biharmonic unit vector field if and only if every $(S, W)$-angle $\theta$ of $V$ satisfies

$$
\widehat{\Delta}(\widehat{\Delta} \theta)-\widehat{\operatorname{div}}\left(\left(k_{g} \circ \pi\right)^{2} \widehat{\operatorname{grad} \theta}\right)=\widehat{\Delta}(\widehat{\operatorname{div}} \widehat{Z})+\widehat{\operatorname{div}}\left(\left(k_{g} \circ \pi\right)^{2} \widehat{Z}\right),
$$

where $\widehat{Z}=(\alpha \circ \pi) \widehat{S}+(b \circ \pi) \widehat{W}$.

(ii) There exists a biharmonic unit vector field in each homotopy class.

Proof (i) Let $X: I \rightarrow \mathfrak{X}^{1}\left(T^{2}\right)$ be a smooth path in $\mathfrak{X}^{1}\left(T^{2}\right)$ such that $X_{0}=V$. We denote by $\theta_{t} \in C^{\infty}\left(\mathbb{R}^{2} \times I\right)$ the angle function for $X_{t}$ and set $\dot{\theta}=\frac{\partial \theta}{\partial_{t}}, \theta_{0}=\theta$. Using (4.6), we have

$$
\begin{aligned}
\frac{\mathrm{d}}{\mathrm{d} t} G\left(\theta_{t}\right)= & 2 \int_{Q}\left\{\left(\widehat{\Delta} \theta_{t}-\widehat{\operatorname{div}} \widehat{Z}\right) \widehat{\Delta} \dot{\theta}+\left(k_{g} \circ \pi\right)^{2} \widehat{g}\left(\widehat{\operatorname{grad} \theta}, \widehat{\operatorname{grad}} \theta_{t}+\widehat{Z}\right)\right\} \pi^{*}\left(\omega_{T}\right) \\
= & 2 \int_{Q}\left\{\widehat{\Delta}\left(\widehat{\Delta} \theta_{t}-\widehat{\operatorname{div}} \widehat{Z}\right) \dot{\theta}+\widehat{\operatorname{div}}\left[\dot{\theta}\left(k_{g} \circ \pi\right)^{2} \widehat{\left(\operatorname{grad} \theta_{t}\right.}+\widehat{Z}\right)\right] \\
& \left.\left.-\dot{\theta} \widehat{\operatorname{div}}\left[\left(k_{g} \circ \pi\right)^{2} \widehat{\left(\operatorname{grad} \theta_{t}\right.}+\widehat{Z}\right)\right]\right\} \pi^{*}\left(\omega_{T}\right) \\
= & \left.2 \int_{Q}\left\{\dot{\theta}\left[\widehat{\Delta}\left(\widehat{\Delta} \theta_{t}-\widehat{\operatorname{div}} \widehat{Z}\right)-\widehat{\operatorname{div}}\left[\left(k_{g} \circ \pi\right)^{2} \widehat{\left(\operatorname{grad} \theta_{t}\right.}+\widehat{Z}\right)\right]\right]\right\} \pi^{*}\left(\omega_{T}\right) .
\end{aligned}
$$

As a consequence,

$$
\left.\left.\frac{\mathrm{d}}{\mathrm{d} t} G\left(\theta_{t}\right)\right|_{t=0}=2 \int_{Q}\left\{\beta\left[\widehat{\Delta}(\widehat{\Delta} \theta-\widehat{\operatorname{div}} \widehat{Z})-\widehat{\operatorname{div}}\left[\left(k_{g} \circ \pi\right)^{2} \widehat{(\operatorname{grad} \theta}+\widehat{Z}\right)\right]\right]\right\} \pi^{*}\left(\omega_{T}\right),
$$

where $\beta(\xi)=\dot{\theta}(\xi, 0)$ for any $\xi \in \mathbb{R}^{2}$. Setting $\beta(\xi)=\widehat{\Delta}(\widehat{\Delta} \theta-\widehat{\operatorname{div}} \widehat{Z})(\xi)-\widehat{\operatorname{div}}\left[\left(k_{g} \circ\right.\right.$ $\left.\left.\pi)^{2} \widehat{(\operatorname{grad} \theta}+\widehat{Z}\right)\right](\xi)$ in (4.8) yields (4.7).

(ii) Fix a homotopy class and consider all solutions $\theta \in \operatorname{Per}(m, n)$. Choose any fixed $\theta_{1} \in \operatorname{Per}(m, n)$, then $\theta-\theta_{1} \in \operatorname{Per}(0,0)$ and by [11, Lemma 3 i)] there is $\alpha \in C^{\infty}\left(T^{2}\right)$ such that $\theta-\theta_{1}=\alpha \circ \pi$. Furthermore, still by [11], there exists $Y \in \mathfrak{X}\left(T^{2}\right)$ such that $\widehat{\operatorname{grad}} \theta_{1}=Y \circ \pi$ and $\widehat{\Delta} \theta_{1}=-\operatorname{div} Y \circ \pi$, so

$$
\widehat{\Delta} \theta=(\Delta \alpha-\operatorname{div} Y) \circ \pi, \widehat{\operatorname{div}}\left(\left(k_{g} \circ \pi\right)^{2} \widehat{\operatorname{grad} \theta}\right)=\operatorname{div}\left(k_{g}^{2}(Y+\operatorname{grad} \alpha)\right) \circ \pi .
$$

Using (4.9), Eq. (4.7) is transformed into

$$
\Delta(\Delta \alpha)-\operatorname{div}\left(k_{g}^{2} \operatorname{grad} \alpha\right)=\Delta(\operatorname{div}(Y+Z))+\operatorname{div}\left(k_{g}^{2}(Y+Z)\right)=f .
$$

With similar arguments as in the proof of Theorem 4.2, we conclude that Eq. (4.10) has a solution if and only if $\int_{T^{2}} f v_{g}=0$, which clearly holds. Denote by $\alpha_{0}$ this solution, then $\theta=\theta_{1}+\alpha_{0} \circ \pi$ is a solution to Eq. (4.10).

Remark 4.2 In general, if $\left(T^{2}, g\right)$ is not flat, the biharmonic unit vector fields obtained in Theorems 4.2 and 4.4 are not harmonic. The solutions obtained in Theorem 4.2(ii) are harmonic unit vector fields (i.e. the sections found by Wiegmink) precisely when the functions $u$ and $\Delta u$ have (pointwise) collinear gradients, as, for example, if $u$ is an eigenfunction of the Laplacian.

Theorem 4.5 On a 2-torus $\left(T^{2}, g\right)$, the Hessian form of $E_{2}$ is positive semi-definite at all critical points $V$ of $E_{2}$, i.e. $\frac{\mathrm{d}^{2}}{\mathrm{~d} t^{2}}\left\{E_{2}(X(t))\right\}_{t=0} \geq 0$ for all $C^{\infty}$-variations $X(t)(t \in(-\epsilon, \epsilon), \epsilon>0)$ of $V$ within $\mathfrak{X}^{1}\left(T^{2}\right)$. 
Proof Differentiating, w.r.t the variable $t$, the first equation in the proof of Theorem 4.4, we get

$$
\begin{aligned}
\frac{\mathrm{d}^{2}}{\mathrm{~d} t^{2}} G\left(\theta_{t}\right)= & 2 \int_{Q}\left\{\ddot{\theta}\left[\widehat{\Delta}\left(\widehat{\Delta} \theta_{t}-\widehat{\operatorname{div}} \widehat{Z}\right)-\widehat{\operatorname{div}}\left[\left(k_{g} \circ \pi\right)^{2}\left(\widehat{\operatorname{grad} \theta_{t}}+\widehat{Z}\right)\right]\right]\right. \\
& \left.+\dot{\theta}\left[\widehat{\Delta}(\widehat{\Delta} \dot{\theta})-\widehat{\operatorname{div}}\left[\left(k_{g} \circ \pi\right)^{2} \widehat{\operatorname{grad} \dot{\theta}}\right]\right]\right\} \pi^{*}\left(\omega_{T}\right) .
\end{aligned}
$$

Evaluating at $t=0$ and using (4.7) (since $V$ is a biharmonic unit vector field), we have

$$
\left.\frac{\mathrm{d}^{2}}{\mathrm{~d} t^{2}} G\left(\theta_{t}\right)\right|_{t=0}=2 \int_{Q}(\widehat{\Delta} \beta)^{2} \pi^{*}\left(\omega_{T}\right)+2 \int_{Q}\left(k_{g} \circ \pi\right)^{2}\|\widehat{\operatorname{grad}} \beta\|_{\hat{g}}^{2} \pi^{*}\left(\omega_{T}\right) \geq 0,
$$

where $\beta(\xi)=\dot{\theta}(\xi, 0)$ for any $\xi \in \mathbb{R}^{2}$.

Remark 4.3 In [11], Wiegmink proved that harmonic unit vector fields on two-dimensional torus $\left(T^{2}, g\right)$ are stable.

\section{References}

1. Baird, P., Wood, J.C.: Harmonic Morphisms Between Riemannian Manifolds, London Mathematical Society Monographs, vol. 29. Oxford University Press, Oxford (2003)

2. Besse, A.: Einstein Manifolds. Springer, Berlin (1987)

3. Dragomir, S., Perrone, D.: Harmonic Vector Fields: Variational Principles and Differential Geometry. Elsevier, Amsterdam (2011)

4. Eells, J., Sampson, J.H.: Harmonic mappings of Riemannian manifolds. Am. J. Math. 86(1), 109-160 (1964)

5. Gil-Medrano, O., González-Dávila, J.C., Vanhecke, L.: Harmonic and minimal invariant unit vector fields on homogeneous Riemannian manifolds. Houston J. Math. 27(2), 377-409 (2001)

6. Markellos, M.K.: The biharmonicity of unit vector fields on the Poincaré half-space $H^{n}$. In: Proceedings of the VIII International Colloquium on Differential Geometry, Santiago de Compostella, World Scientific, pp. 247-256 (2009)

7. Markellos, M., Urakawa, H.: The bienergy of unit vector fields. Ann. Glob. Anal. Geom. 46, 431-457 (2014)

8. Markellos, M., Urakawa, H.: The biharmonicity of sections of the tangent bundle. Monatsh. Math. 178, 389-404 (2015)

9. Milnor, J.: Curvature of left invariant metrics on Lie groups. Adv. Math. 21, 293-329 (1976)

10. Ou, Y.L., Wang, Z.P.: Constant mean curvature and totally umbilical biharmonic surfaces in 3-dimensional geometries. J. Geom. Phys. 61, 1845-1853 (2011)

11. Wiegmink, G.: Total bending of vector fields on Riemannian manifolds. Math. Ann. 303, 325-344 (1995)

12. Wood, C.M.: On the energy of a unit vector field. Geom. Dedicata 64, 319-330 (1997)

Publisher's Note Springer Nature remains neutral with regard to jurisdictional claims in published maps and institutional affiliations. 